$07 ; 14$

\title{
Адаптивные свойства спайковых нейроморфных сетей с синаптическими связями на основе мемристивных элементов
}

\author{
(C) К.Э. Никируй ${ }^{1,2}$, А.В. Емельянов ${ }^{1,2}$, В.В. Рыльков ${ }^{1,3}$, А.В. Ситников ${ }^{1,4}$, В.А. Демин ${ }^{1,2,5}$ \\ ${ }^{1}$ Национальный исследовательский центр „Курчатовский институт“, Москва, Россия \\ ${ }^{2}$ Московский фризико-технический институт (Государственный университет), Долгопрудный, Московская обл., Россия \\ ${ }^{3}$ Фрязинский филиал Института радиотехники и электроники им. В.А. Котельникова РАН, Фрязино, Московская обл., \\ Россия \\ ${ }^{4}$ Воронежский государственный технический университет, Воронеж, Россия \\ ${ }^{5}$ Нижегородский государственный университет им. Н.И. Лобачевского, Нижний Новгород, Россия \\ E-mail: NikiruyKristina@gmail.com
}

Поступило в Редакцию 25 января 2019г.

В окончательной редакции 25 января 2019г.

Принято к публикации 28 января 2019 г.

\begin{abstract}
Нейроморфные вычислительные сети (НВС) с синаптическими связями на основе мемристоров („сопротивления с эффектом памяти“) могут обеспечить гораздо более эффективный подход при аппаратной реализации различных типов алгоритмов нейронных сетей, чем традиционные элементы на основе комплементарной технологии. Для реализации эффективных НВС необходимо, чтобы сопротивление мемристора могло быть изменено по локальным правилам (например, пластичности, зависящей от времени прихода импульсов, - STDP). Изучена возможность локального обучения мемристоров на основе нанокомпозита $\left(\mathrm{Co}_{0.4} \mathrm{Fe}_{0.4} \mathrm{~B}_{0.2}\right)_{x}\left(\mathrm{LiNbO}_{3}\right)_{1-x}$ по правилам STDP. На примере $\mathrm{HBC}$, состоящей из четырех входных и одного выходного нейрона, продемонстрирована возможность ее обучения по правилам STDP. Обнаружено, что конечное состояние НВС не зависит от начального состояния данной сети, а зависит только от условий обучения (последовательности импульсов). Изучена зависимость результата обучения от значения порогового тока выходного нейрона. Полученные результаты открывают перспективы создания автономных HBC, способных к обучению решению сложных когнитивных задач.
\end{abstract}

DOI: 10.21883/PJTF.2019.08.47615.17712

В последнее время существенно возрос интерес к аппаратной разработке нейроморфных вычислительных сетей (НBC) на базе мемристоров благодаря их высокой производительности при малом энергопотреблении для решения так называемых антропоморфных задач (распознавание образов и речи, обобщение, принятие решений, прогнозирование и т.д.) [1]. Нейроны в таких НВС моделируются либо программно, либо аппаратно с использованием КМОП-микросхем [2], тогда как связи между нейронами (синапсы) моделируются особыми резистивными элементами (мемристорами), способными под действием электрических импульсов квазинепрерывно изменять и сохранять свое сопротивление в некотором окне между высокоомным $\left(R_{o f f}\right)$ и низкоомным $R_{\text {on }}$ резистивными состояниями [3]. Заметим, что многоуровневый характер резистивного переключения (РП) мемристоров играет ключевую роль в реализации НВС и присущ не всем типам мемристоров. Однако многие из них обладают малым энергопотреблением при записи/считывании информации $(\sim 10 \mathrm{fJ})$, энергонезависимостью и высоким быстродействием ( $1 \mathrm{~ns})$ и потому перспективны для создания универсальной памяти [4].

Отличительными особенностями НВС с мемристивными синаптическими связями (по сравнению с обычными вычислительными системами, базирующимися на архитектуре фон Неймана) являются их архитектура и возможность обучения (как с учителем, так и без него) на основе различных правил, которые в настоящее время представляют собой предмет интенсивных дискуссий и исследований. Можно выделить два основных направления этих исследований:

1) перенос алгоритмов обучения программных нейронных сетей на аппаратную основу (реализация сетей типа перцептрона [3,5,6], сверточных сетей [7] и др.);

2) использование так называемых биологически правдоподобных алгоритмов обучения сетей [8].

Недостаток первого подхода связан со сложностью его аппаратной реализации, поскольку на каждом шаге обучения необходимо не только знать с высокой точностью текущее состояние каждого синаптического веса мемристора (его резистивное состояние), но и иметь возможность изменить его на строго определенную величину [9]. Поэтому данный подход обучения $\mathrm{HBC}$, скорее всего, подходит для решения определенных (протоколированных) задач с использованием внешнего учителя. Второй подход, основанный на биологически правдоподобных принципах обучения, мало изучен с практической точки зрения. Считается, однако, что он может иметь хорошие перспективы для построения самообучаемых НВС. В биологических нейронных системах обучение происходит благодаря способности синапсов изменять свой вес, которая называется синаптической пластичностью [10]. Для описания эволюции 

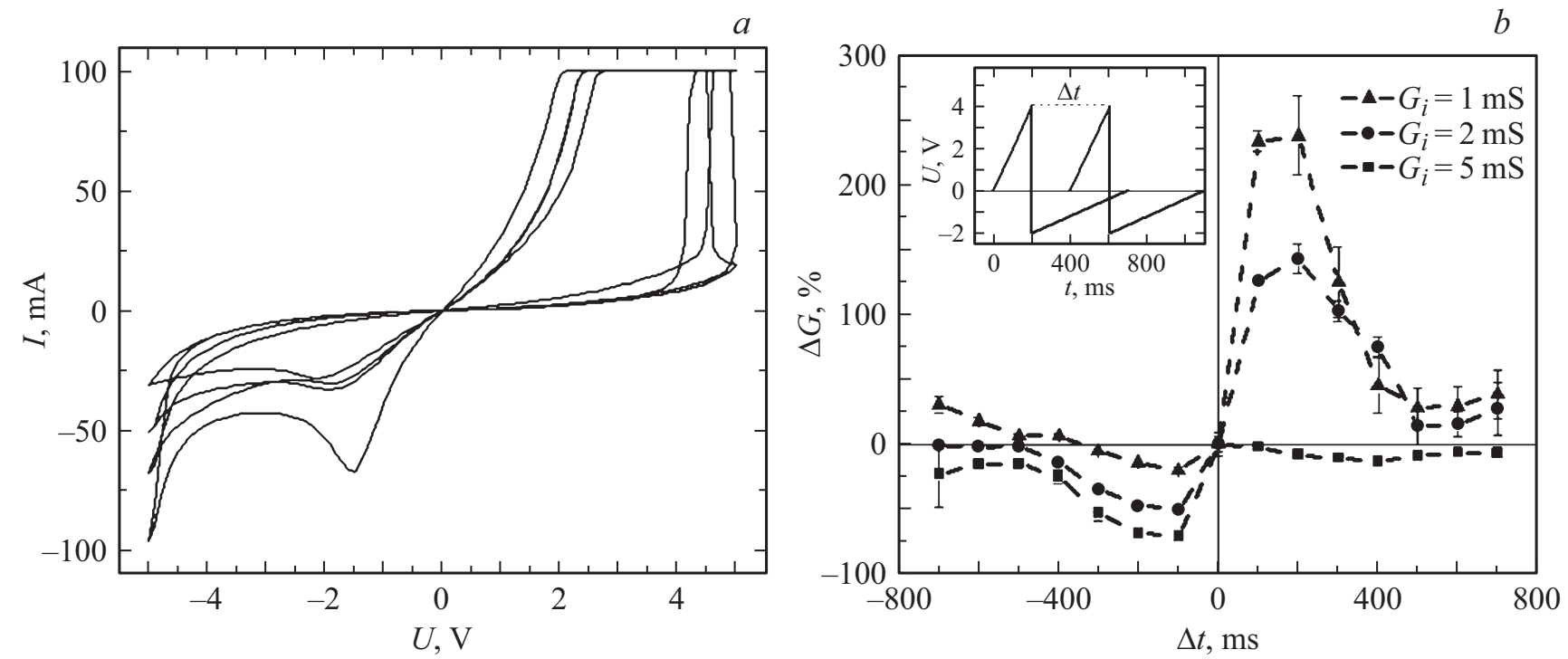

Рис. 1. $a$ - типичные BAX четырех НК мемристоров; $b$ - окно STDP для трех различных начальных состояний: 1,2 и 5 mS. На вставке - пример пре- и постсинаптических спайков.

синаптических весов привлекаются такие механизмы, как долго- и кратковременная потенциация и депрессия [11], флуктуация парных импульсов [12] и др. В качестве одного из перспективных механизмов рассматривается механизм пластичности, зависящей от времени прихода импульсов (spike timing dependent plasticity, STDP) [10]. Согласно STDP, синаптический вес увеличивается в случае, если постсинаптический нейрон генерирует импульс (спайк) сразу после пресинаптического, что указывает на наличие причинно-следственной связи, и наоборот. Механизм изменения мемристивного веса по правилу STDP был продемонстрирован на примере некоторых мемристоров [10,13-16] и использован в спайковых HBC [17].

Перспективными для нейроморфных приложений являются мемристивные структуры на основе сегнетоэлектрика $\mathrm{LiNbO}_{3}$ [18]. Нами показано [19], что при введении наночастиц сплава $\mathrm{Co}_{0.4} \mathrm{Fe}_{0.4} \mathrm{~B}_{0.2}$ в матрицу $\mathrm{LiNbO}_{3}$ и формировании мемристивных структур металл/нанокомпозит/металл $(M / \mathrm{HK} / M)$ на основе НК $\left(\mathrm{Co}_{0.4} \mathrm{Fe}_{0.4} \mathrm{~B}_{0.2}\right)_{x}\left(\mathrm{LiNbO}_{3}\right)_{1-x}$ удается существенно увеличить число стабильных циклов РП: с 500 циклов [18] до более $10^{5}$ раз при отношении $R_{o f f} / R_{\text {on }}$ более 50 . При этом в данном окне сопротивлений обеспечивается возможность прецизионного задания резистивного состояния с точностью не хуже $0.5 \%$, что свидетельствует о высокой пластичности структур $M / \mathrm{HK} / M$ [20]. Цель настоящей работы заключается в изучении возможности обучения структур $M / \mathrm{HK} / M$ по правилам STDP, а также в построении и исследовании особенностей функционирования простейшей спайковой НВС на основе мемристивных структур $M / \mathrm{HK} / M$.

Изучались мемристоры конденсаторного типа $M / \mathrm{HK} / M$ на базе $\mathrm{HK}\left(\mathrm{Co}_{0.4} \mathrm{Fe}_{0.4} \mathrm{~B}_{0.2}\right)_{x}\left(\mathrm{LiNbO}_{3}\right)_{1-x} \quad \mathrm{c}$ $x \approx 0.1$, синтезированные методом ионно-лучевого распыления на ситалловых подложках [19]. В качестве металла выступала трехслойная структура $\mathrm{Cr} / \mathrm{Cu} / \mathrm{Cr}$ (размер верхнего электрода составлял $0.5 \times 0.2 \mathrm{~mm}$ ). Электрофизические исследования структур $M / \mathrm{HK} / M$, включая измерения вольт-амперных характеристик $(\mathrm{BAX})$, выполнялись с помощью четырехканального источника-измерителя PXIe-4140 (National Instruments) на аналитической зондовой станции PM5 (Cascade Microtech).

Особенностью мемристоров при РП является наличие характерного гистерезиса, наблюдаемого на ВАХ. На рис. 1, $a$ представлены типичные BAX четырех различных НК мемристоров, которые измерены при заземленном нижнем электроде и развертке напряжения смещения $U$ верхнего электрода по линейному закону в последовательности от $0 \rightarrow+U_{0} \rightarrow 0 \rightarrow-U_{0} \rightarrow 0$ с шагом $0.1 \mathrm{~V}$ и длительностью $50 \mathrm{~ms} ; U_{0}=5 \mathrm{~V}$. Из рисунка видно, что параметры РП различных мемристоров различаются несущественно. Это свидетельствует о хорошей воспроизводимости устройств при выбранной технологии изготовления. Данные мемристоры использованы в дальнейшем для построения НВС.

Для создания спайковой НВС необходима проверка возможности обучения отдельных НК мемристоров по правилам STDP. Для этого нижний электрод был использован как пресинаптический вход, а верхний как постсинаптический. Мы применяли одинаковые преи постсинаптические спайки (вставка на рис. $1, b$ ). Амплитуда спайка была выбрана таким образом, что сам по себе спайк не изменял сопротивление мемристора. Однако если два спайка с малой задержкой между ними по времени $\Delta t$ будут поданы на мемристор, падение напряжения на нем может оказаться достаточным для 

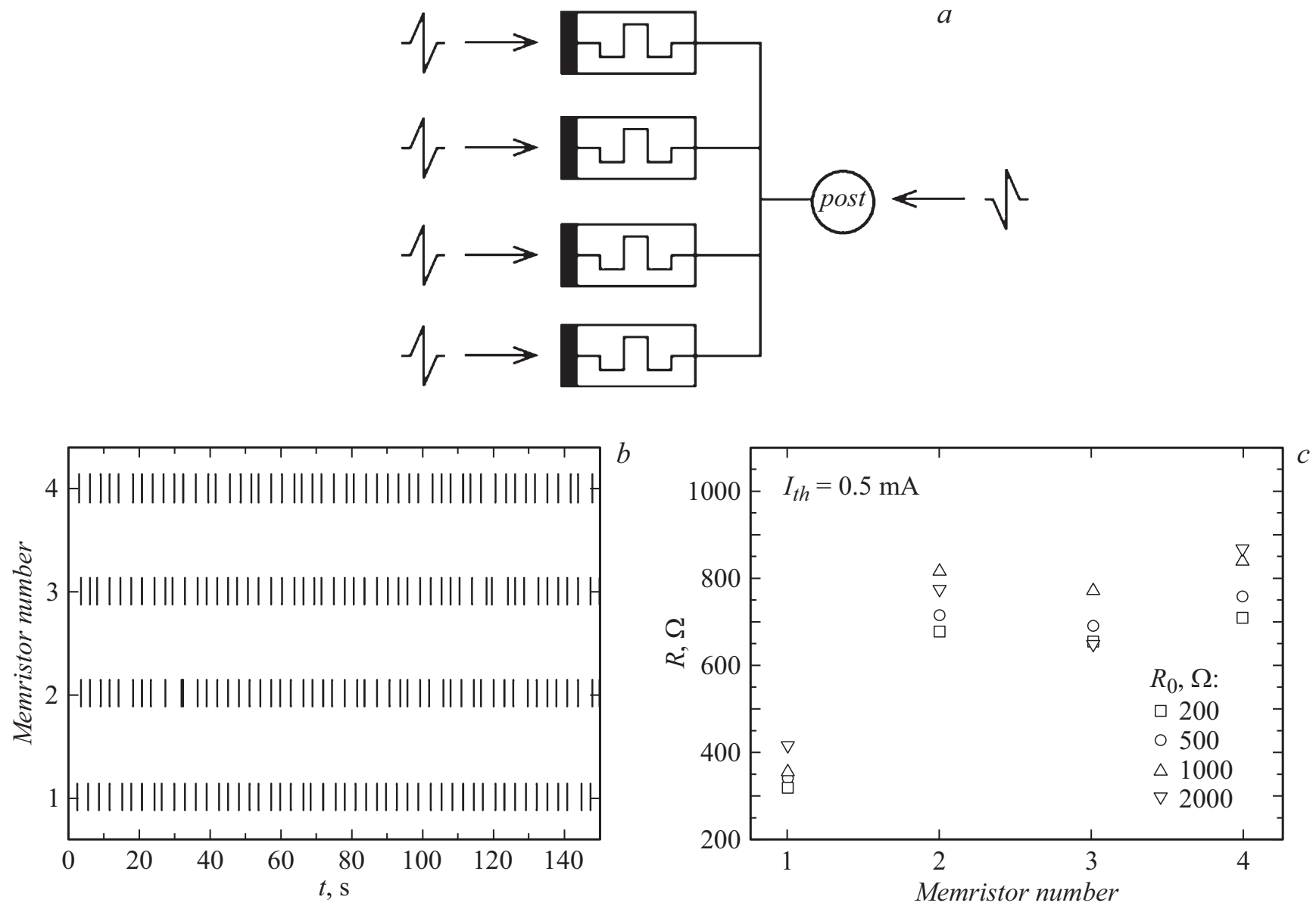

Рис. 2. $a-$ схематическое изображение построенной НВС; $b-$ последовательности пресинаптических спайков; $c-$ распределение конечных значений резистивных состояний мемристоров после обучения $\left(I_{t h}=0.5 \mathrm{~mA}\right)$, символы различной формы соответствуют разным начальным состояниям мемристоров.

изменения его резистивного состояния. Проводимость $G$ мемристора можно связать с синаптическим весом, тогда изменение проводимости $\Delta G$ будет эквивалентно изменению синаптического веса. Измерения проводимости осуществлялись до и после подачи последовательности пре- и постсинаптических спайков. Зависимость относительного изменения веса от времени $\Delta t$ (окно STDP) для различных начальных состояний представлено на рис. $1, b$. Каждое значение бралось как медианное по десяти измерениям. Из рис. $1, b$ видно, что синаптическая потенция $(\Delta G>0)$ происходит при $\Delta t>0$, депрессия $(\Delta G<0)-$ при $\Delta t<0$. Подобный вид зависимости был обнаружен и в биологических системах [21]. Следует отметить, что обучение по STDP зависит от начального резистивного состояния мемристора. Если состояние близко к „крайним“ резистивным состояниям $R_{o n}$ или $R_{o f f}$ (квадраты и треугольники на рис. $1, b)$, то изменение синаптического веса будет асимметрично: оно будет сильнее проявляться только в одном из квадрантов (I или III). Для промежуточного значения (кружки на рис. $1, b$ ) изменение веса более симметрично по квадрантам I и III. Такая зависимость от начального состояния может быть объяснена конечными значениями проводимости мемристора. Обнаруженная зависимость изменения веса мемристивных структур $M / \mathrm{HK} / M$ по STDP позволяет рассматривать их как перспективные элементы для автономных спайковых НBC.

Для изучения особенностей обучения с использованием STDP была разработана HBC, состоящая из четыpex пресинаптических нейронов, соединенных с постсинаптическим нейроном посредством мемристивных синапсов, как показано на рис. $2, a$. Все нейроны были программными: пресинаптические нейроны были запрограммированы генерировать различные последовательности спайков (рис. $2, b)$; форма спайков показана на вставке к рис. $1, b$. Отклонения времени пресинаптических спайков от среднего (при частоте $\sim 0.5 \mathrm{~Hz}$ ) были распределены по Пуассону (рис. $2, b$ ) и запоминались для каждого нейрона. Постсинаптический нейрон являлся пороговым (генерировал спайк в том случае, когда суммарная величина тока превышала пороговое значение $\left.I_{t h}\right)$. Изначально все мемристоры НВС приводились к заданному состоянию $R_{0}$ при помощи специально разработанного 


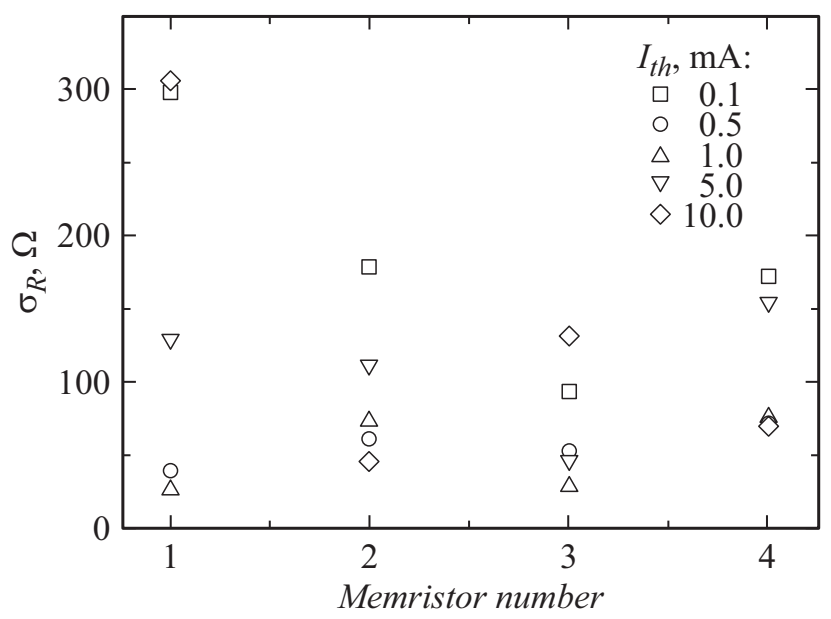

Рис. 3. Среднеквадратичное отклонение конечного сопротивления при различных значениях порогового тока для каждого мемристора.

алгоритма [20]. В качестве $R_{0}$ были выбраны значения 200, 500, 1000, $2000 \Omega$. После этого с пресинаптических нейронов подавалась запомненная последовательность спайков, которая всегда была одинаковой для различных $R_{0}$ (рис. $\left.2, b\right)$. Далее измерялись конечные сопротивления мемристоров. Из рис. 2, $c$ видно, что итоговое резистивное состояние системы зависит только от условий обучения (в нашем случае от последовательности подаваемых импульсов) и практически не зависит от начальных значений $R_{0}$. Таким образом, обучение HBC по правилам STDP демонстрирует адаптивные свойства, что может быть использовано при создании НВС с обучением „без учителя“. Отметим, что при изменении значения порогового тока результат обучения может сильно измениться. Обучение при очень малых $(<0.1 \mathrm{~mA})$ и очень больших (> $5 \mathrm{~mA})$ пороговых токах приводит к значительному увеличению среднеквадратичного отклонения итогового резистивного состояния системы (рис. 3). Оптимальными значениями пороговых токов являются $0.5-1 \mathrm{~mA}$.

Таким образом, показано, что конечное состояние HBC, обученной с использованием правил STDP, не зависит от начального состояния данной сети в случае выбора оптимального порогового тока нейрона, а зависит только от условий обучения (последовательности импульсов). Данное обстоятельство делает обучение по правилам STDP основой для автономного обучения („без учителя“) нейроморфных сетей на основе мемристивных связей и открывает перспективы использования данных правил при решении сложных когнитивных задач.

Измерения выполнены на оборудовании Ресурсного центра электрофизических методов при поддержке Курчатовского комплекса НБИКС-природоподобных технологий (Приказ № 1713).
Работа выполнена при финансовой поддержке Российского научного фонда (№ 18-79-10253) в части изучения отдельных мемристоров и их обучения по STDP и гранта Правительства РФ № 074-02-2018-330(2) в части исследования адаптивных свойств на шумовом сигнале нейрона с мемристивными весами.

\section{Список литературы}

[1] Wang J., Zhuge F. // Adv. Mater. Technol. 2019. P. 1800544. First published: 03 January 2019.

[2] Sourikopoulos I., Hedayat S., Loyez C., Danneville F., Hoel V., Mercier E., Cappy A. // Front. Neurosci. 2017. V. 11. P. 123.

[3] Li C., Hu M., Li Y., Jiang H., Ge N., Montgomery E., Zhang J., Song W., Dávila N., Graves C.E., Li Z., Strachan J.P., Lin P., Wang Z., Barnell M., Wu Q., Williams R.S., Yang J.J., Xia Q. // Nature Electron. 2018. V. 1. P. 52-59.

[4] Ielmini D. // Semicond. Sci. Technol. 2016. V. 31. P. 063002.

[5] Prezioso M., Merrikh-Bayat F., Hoskins B.D., Adam G.C., Likharev K.K., Strukov D.B. // Nature. 2015. V. 521. P. 6164.

[6] Антонов И.Н., Белов А.И., Михайлов А.Н., Морозов О.А., Овчинников П.Е. // Радиотехника и электроника. 2018. T. 63. № 8. C. 880-888.

[7] Acharya U.R., Oh S.L., Hagiwara Y., Tan J.H., Adeli H. // Comput. Biol. Med. 2018. V. 100. P. 270-278.

[8] Pedretti G., Milo V., Ambrogio S., Carboni R., Bianchi S., Calderoni A., Ramaswamy N., Spinelli A.S., Ielmini D. // Sci. Rep. 2017. V. 7. P. 5288.

[9] Emelyanov A.V., Lapkin D.A., Demin V.A., Erokhin V.V., Battistoni S., Baldi G., Dimonte A., Korovin A.N., Iannotta S., Kashkarov P.K., Kovalchuk M.V. // AIP Adv. 2016. V. 6. P. 111301.

[10] Prezioso M., Merrikh-Bayat F., Hoskins B.D., Likharev K., Strukov D.B. // Sci. Rep. 2016. V. 6. P. 21331.

[11] Kim K.-H., Gaba S., Wheeler D., Cruz-Albrecht J., Hussain T., Srinivasa N., Lu W. // Nano Lett. 2012. V. 12. P. 389-395.

[12] Hu S.G., Liu Y., Chen T.P., Liu Z., Yu Q., Deng L.J., Yin Y., Hosaka S. // Appl. Phys. Lett. 2013. V. 102. P. 183510.

[13] Serb A., Bill J., Khiat A., Berdan R., Legenstein R., Prodromakis T. // Nature Commun. 2016. V. 7. P. 12611.

[14] Hsieh C.C., Roy A., Chang Y.F., Shahrjerdi D., Banerjee S.K. // Appl. Phys. Lett. 2016. V. 109. P. 223501.

[15] Lapkin D.A., Emelyanov A.V., Demin V.A., Berzina T.S., Erokhin V.V. // Microelectron. Eng. 2018. V. 185-186. P. 4347.

[16] Hansen M., Zahari F., Kohlstedt H., Ziegler M. // Sci. Rep. 2018. V. 8. P. 8914.

[17] Wang Z., Joshi S., Savel'ev S., Song W., Midya R., Li Y., Rao M., Yan P., Asapu S., Zhuo Y., Jiang H., Lin P., Li C., Yoon J.H., Upadhyay N.K., Zhang J., Hu M., Strachan J.P., Barnell M., Wu Q., Wu H., Williams R.S., Xia Q., Yang J.J. // Nature Electron. 2018. V. 1. P. 137-145.

[18] Pan X., Shuai Y., Wu C., Luo W., Sun X., Zeng H., Zhou S., Bïtger R., Ou X., Mikolajick T., Zhang W., Schmidt H. // Appl. Phys. Lett. 2016. V. 108. P. 032904. 
[19] Рыльков В.В., Николаев С.Н., Демин В.А., Емельянов А.В., Ситников А.В., Никируй К.Э., Леванов В.А., Пресняков М.Ю., Талденков А.Н., Васильев А.Л., Черноглазов К.Ю., Веденеев А.С., Калинин Ю.Е., Грановский А.Б., Тугушев В.В., Бугаев А.С. // ЖЭТФ. 2018. Т. 153. В. 3. C. $424-441$.

[20] Никируй К.Э., Емельянов А.В., Демин В.А., Рыльков В.В., Ситников А.В., Кашкаров П.К. // Письма в ЖТФ. 2018. T. 44. B. 10. C. 20-28.

[21] Hennequin G., Agnes E.J., Vogels T.P. // Annu. Rev. Neurosci. 2017. V. 40. P. 557-579. 